\title{
Protective effect of 3-n-butylphthalide against hypertensive nephropathy in spontaneously hypertensive rats
}

\author{
JUN ZHU, YANTAO ZHANG and CHANGHAI YANG \\ Department of Urology, Tianjin Medical University General Hospital, Tianjin 300052, P.R. China
}

Received April 2, 2014; Accepted July 22, 2014

DOI: $10.3892 / \mathrm{mmr} .2014 .2791$

\begin{abstract}
Previous studies have demonstrated that a natural product of celery seeds, 3-n-butylphthalide (NBP), has significant antihypertensive effects that are widely utilized in Chinese traditional medicine. The present study aimed to investigate the effects of NBP on hypertensive nephropathy, as well as the mechanisms underlying this disease in spontaneously hypertensive rats (SHRs). SHRs were treated orally with saline, NBP (15 or $30 \mathrm{mg} / \mathrm{kg}$ ) or losartan $(10 \mathrm{mg} / \mathrm{kg}$ ) daily for 20 weeks, during which time blood pressure was measured every four weeks. At the end of the 20 -week treatment, blood and urine samples were collected for biochemical analysis, and kidney tissues were obtained for histopathological analysis and immunohistochemistry. Enzyme-linked immunosorbent assays and western blotting were used to analyze the expression of transforming growth factor (TGF)- $\beta 1$ in blood and kidney tissues, respectively. The results showed that NBP effectively attenuated progression of hypertensive nephropathy by decreasing urinary albumin excretion and blood urea nitrogen levels. It significantly decreased blood pressure (although less markedly than losartan) and the incidence of glomerulosclerosis. In addition, it alleviated tubular impairment and significantly decreased oxidative stress, as well as the expression of pro-inflammatory cytokines and TGF- $\beta 1$ in kidney tissues. In conclusion, the results suggested that NBP may slow the progression of hypertensive nephropathy by a variety of mechanisms.
\end{abstract}

\section{Introduction}

Hypertension is an important risk factor in the pathogenesis of hypertensive renal injury, which is one of the leading causes of chronic kidney disease worldwide (1-4). Analysis of epidemiological data from dialysis registries in Japan and the USA (5-7) has shown that the incidence of end-stage renal

Correspondence to: Dr Zhu Jun, Department of Urology, Tianjin Medical University General Hospital, 154 Anshan Road, Tianjin 300052, P.R. China

E-mail: zhujun1774@163.com

Key words: hypertension, 3-n-butylphthalide, nephropathy, angiotensin II, angiotensin converting enzyme disease due to hypertensive nephrosclerosis is increasing in a number of countries. It is therefore crucial to identify novel preventive and therapeutic compounds for use in hypertensive renal disease $(8,9)$.

3-n-Butylphthalide (NBP) is a compound extracted from Chinese celery and is used as an antihypertensive herbal traditional Chinese medicine in the treatment of stroke patients $(10,11)$. Several mechanisms may underlie its therapeutic effects. NBP can promote the nitric oxide (NO) production of endothelial cells, resulting in vasodilative effects (12). In addition, NBP can increase the number of cerebral microvessels via upregulation of the expression of vascular endothelial growth factor and hypoxia inducible factor-1 $\alpha$ (13). A randomized, double-blind placebo-controlled trial involving 573 patients in China has shown that NBP is safe and effective for patients with acute noncardioembolic ischemic stroke, particularly for those cases of moderate severity (14). One study demonstrated that NBP decreased blood pressure (BP) and increased heart rate (HR) in hypertensive rats, but had no effect on normotensive rats (10). Furthermore, NBP protects endothelial cells in the microvessels from oxidative and nitrosative stress, mitochondrial damage and subsequent cell death, following oxygen and glucose deprivation in vitro. These are also important risk factors for chronic renal dysfunction induced by hypertension $(12,15,16)$. It was hypothesized that NBP may have a protective effect against the development of hypertensive nephropathy. The current study established a chronic renal injury model, induced by long-term hypertension, to examine the effect of NBP on this process.

Spontaneously hypertensive rats (SHRs) have been widely used as a primary hypertension animal model, in which the hypertensive nephropathy is characterized by multiple renal structural and functional alterations. It has been found that the mechanism underlying renal injury in SHRs comprises a complex pathological network, involving renin, angiotensin II (Ang II), monocytes, macrophages, inflammatory cytokines and oxidative stress $(17,18)$. Generally, in SHRs older than six months, there is automatic progression into severe renal injury characterized by marked proteinuria, elevation of serum creatinine (Scr) and blood urea nitrogen (BUN), reduced creatinine clearance ratio $(\mathrm{CCr})$, glomerulosclerosis, interstitial fibrosis and renal vascular arteriosclerosis. These characteristics render SHRs a good animal model of human hypertensive nephropathy (19).

Previous studies have shown that the use of renin-angiotensin system (RAS) inhibitors, such as Ang-converting 
enzyme inhibitors and Ang receptor blockers, can effectively suppress the progression of established renal disease $(20,21)$. The present study used losartan as a positive control to compare the renal protective effect of NBP on SHRs. It aimed to investigate whether NBP alleviates chronic kidney injury under hypertensive conditions.

\section{Materials and methods}

Ethical considerations. This study was approved by the Institutional Animal Care Committee of Tianjin Medical University General Hospital (Tianjin, China), and was conducted in accordance with the US National Institute of Health Guide for the Care and Use of Laboratory Animals.

Animal treatment protocol. Male spontaneously hypertensive rats (aged 16 weeks and weighing 300-340 g) and normal male Wistar rats (aged 16 weeks and weighing 300-340 g), were obtained from the Institute of Laboratory Animal Science at the Chinese Academy of Medical Sciences (Beijing, China). They were kept in a specific-pathogen-free facility under constant temperature $\left(22 \pm 2^{\circ} \mathrm{C}\right)$ conditions, with $12 \mathrm{~h}$ (7am to $\left.7 \mathrm{pm}\right)$ illumination, and provided with food and water ad libitum.

The hypertensive rats were randomized into four groups ( $\mathrm{n}=10$ per group). These were: Saline control; $10 \mathrm{mg} / \mathrm{kg}$, per oral (p.o.) losartan treatment; $15 \mathrm{mg} / \mathrm{kg}$, p.o. NBP treatment; and $30 \mathrm{mg} / \mathrm{kg}$ p.o. NBP treatment. NBP was purchased from CSPC-NBP Pharmaceutical Co., Ltd. (Shijiazhuang, China) and losartan was purchased from Merck Co., Inc. (Darmstadt, Germany). Losartan and NBP were diluted in sodium carboxymethycellulose-Na. The treatment lasted for up to 20 weeks, during which time blood pressure was measured every four weeks by tail-cuff plethysmography (BP-98A; Softron, Tokyo, Japan) with prior training of investigators to minimize variability in the blood pressure measurement.

Biochemical analyses of blood and urine. At the end of the designated treatments, blood was sampled through the eyes under anesthesia with diethyl ether (30\% concentration; Beihua Ltd, Beijing, China). Continuous collection of urine samples for $24 \mathrm{~h}$ was performed in each animal after placement in metabolic cages (Zhenghua Ltd, Hefei, China) the day prior to blood sample collection. BUN, Scr and urinary albumin were measured by the standard biochemical kits (BHKT Clinical Reagent Co., Ltd., Beijing, China). Creatinine clearance (CCr) was calculated according to the following formula: $\mathrm{CCr}=$ Urinary creatinine $(\mathrm{mg} / \mathrm{ml}) \times$ urine volume $(\mathrm{ml} / \mathrm{kg}) /$ creatinine in plasma (mg/ml) (22).

\section{Histological examination.}

Section preparation. The kidneys were fixed in $10 \%$ phosphate-buffered formalin solution (PBS), and embedded in paraffin. Sections of $2 \mu \mathrm{m}$ thickness were cut and stained with hematoxylin and eosin (H\&E) and Periodic acid-Schiff (PAS). In order to assess the degree of kidney injury, the following semi-quantitative scores were obtained using light microscopy (Eclipse Ni-E, Nikon, Tokyo, Japan).

Glomerular sclerosis score(H\&EandPASstain).Glomerular sclerosis was determined as previously described (22): Grade 0 , no sclerosis; grade $1,<25 \%$ of the glomerulus; grade $2,26-50 \%$ of the glomerulus; grade $3,51-75 \%$ of the glomerulus; and grade $4,76-100 \%$ of the glomerulus. Thirty glomeruli were observed from each specimen under a microscope with x200 magnification. The score of a biopsy was calculated with the following equation: [(number grade 1 glomeruli $)+(2 \times$ number grade 2 glomeruli $)+(3 \times$ number grade 3 glomeruli $)+(4 x$ number grade 4 glomeruli)] x 100 / total number of glomeruli examined (23).

Tubulointerstitial damage. A scoring system was applied, ranging from 0 to 4 , in which tubular atrophy, dilation, casts, interstitial inflammation and fibrosis were assessed in 10 kidney fields at a magnification of $\mathrm{x} 200$. The scoring was as follows: 0 , normal; 1 , lesions in $<25 \%$ of the area; 2 , lesions in $25-50 \%$ of the area; 3 , lesions in $>50 \%$ of the area; and 4 , lesions involving the entire area $(24,25)$.

Immunohistochemistry. Paraffin-embedded sections were subjected to immunohistochemical assays as previously reported (26). Briefly, the sections were deparaffinized with xylene, rehydrated through a graded series of ethanol to water, and then incubated in blocking solution (PBS plus $1 \%$ bovine serum) at room temperature for $1 \mathrm{~h}$. The sections were then incubated overnight at $4^{\circ} \mathrm{C}$ with one of the following primary antibodies: Monoclonal antibody against rat tumor necrosis factor (TNF)- $\alpha$ (1:50 dilution; R\&D Systems, Minneapolis, MN, USA), interleukin (IL)-6 (1:100 dilution; Santa Cruz Biotechnology, Santa Cruz, CA, USA) or goat polyclonal anti-nuclear factor (NF)- $\kappa$ B p65 antibody that detects the endogenous level of total NF- $\kappa \mathrm{B}$ p65 (1:150 dilution; Santa Cruz Biotechnology). All antibodies were diluted with blocking solution. Negative controls consisted of histological sections incubated with PBS rather than the primary antibody. Immunostaining was conducted with an avidinbiotin-peroxidase complex kit (Zhongshan Ltd., Beijing, China) and counterstained with hematoxilin.

Enzyme-linked immunosorbent assay (ELISA) and western blot analysis. Plasma transforming growth factor (TGF)- $\beta 1$ levels were measured by a Quantikine ELISA kit according to the manufacturer's instructions. Briefly, a monoclonal antibody specific for rat TGF- $\beta 1$ (MB100B; R\&D systems, Minneapolis, MN USA) was pre-coated onto a microplate. The standards and test samples were then pipetted into the wells to allow binding of TGF- $\beta 1$ to the immobilized antibodies. After washing away any unbound substances, an enzyme-linked polyclonal antibody specific for TGF- $\beta 1$ was added to the wells (antibody contained within Quantikine ELISA kit MB100B; R\&D systems). Following a wash that removed any unbound antibody-enzyme reagent, a substrate solution (contained within Quantikine ELISA kit MB100B; R\&D systems) was added to the wells and color was developed in proportion to the quantity of TGF- $\beta 1$ bound to the well. The optical density of each well was measured at a wave length of $450 \mathrm{~nm}$ by a microreader (S190, Molecular Devices, Sunnyvale, CA, USA).

TGF- $\beta 1$ expression in renal tissues was examined by western blotting. Renal tissue lysate was prepared using a lysis buffer (Applygen Technologies Inc, Beijing, China) containing $25 \mathrm{mmol} / \mathrm{l}$ Tris- $\mathrm{HCl}, 150 \mathrm{mmol} / \mathrm{l} \mathrm{NaCl}, 5 \mathrm{mmol} / \mathrm{l}$ ethyleneglycol bis(2-aminoethyl ether)tetraacetic acid, $5 \mathrm{mmol} / \mathrm{l}$ ethylenediamine tetraacetic acid, $10 \mathrm{mmol} / \mathrm{l}$ sodium fluoride, $1 \mathrm{mmol} / 1$ phenylmethyl sulfonylfluoride, 
Table I. BUN, CCr and UAE values of SHRs after 20 weeks of treatment with saline, losartan or NBP.

\begin{tabular}{lcccr}
\hline Parameter & SHR-saline & Losartan $(10 \mathrm{mg} / \mathrm{kg})$ & NBP $(15 \mathrm{mg} / \mathrm{kg})$ & NBP $(30 \mathrm{mg} / \mathrm{kg})$ \\
\hline Mice, $\mathrm{n}$ & 10 & 10 & 10 & 10 \\
$\mathrm{UAE}(\mathrm{mg} / \mathrm{ml} /$ day $)$ & $25.76 \pm 7.25$ & $18.11 \pm 5.66^{\mathrm{a}}$ & $20.08 \pm 10.04^{\mathrm{a}}$ & $17.52 \pm 4.46^{\mathrm{a}}$ \\
$\mathrm{BUN}(\mathrm{mg} / \mathrm{dl})$ & $23.69 \pm 1.64$ & $20.45 \pm 1.32^{\mathrm{a}}$ & $20.06 \pm 0.95^{\mathrm{a}}$ & $19.84 \pm 2.15^{\mathrm{a}}$ \\
$\mathrm{CCr}(\mathrm{ml} / \mathrm{min} /$ & $0.79 \pm 0.35$ & $1.19 \pm 0.37^{\mathrm{a}}$ & $0.09 \pm 0.16$ & $1.15 \pm 0.12^{\mathrm{a}}$ \\
100 g body weight $)$ & & & &
\end{tabular}

${ }^{\mathrm{a}} \mathrm{P}<0.05$, compared with the SHR-saline group. BUN, blood urea nitrogen; $\mathrm{CCr}$, creatinine clearance; UAE, urinary albumin excretion; SHR, spontaneously hypertensive rats.

$1 \%$ TritonX-100, 0.5\% Nonidet P40, $10 \mathrm{mg} / 1$ aprotinin and $10 \mathrm{mg} / \mathrm{l}$ leupeptin, and quantified by the Bradford dye-binding procedure (Applygen Technologies Inc.). Equal quantities of protein were separated by SDS-PAGE $(5 \%$ stacking gel and $10 \%$ separating gel for $\beta$-actin or $12 \%$ separating gel for TGF- $\beta 1$ ) and electroblotted onto nitrocellulose membranes (Merck Millipore, Darmstadt, Germany). After blocking with $3 \%$ bovine serum albumin, the membranes were incubated with rabbit anti-rat monoclonal TGF- $\beta 1$ (Cell Signaling Technology, Inc., Beverly, MA, USA) and mouse anti-rat polyclonal $\beta$-actin (Santa Cruz Biotcehnology, Inc.) antibodies overnight. Membranes were then incubated with a monoclonal horseradish peroxidase-conjugated goat antirabbit secondary antibody (ZSGB-BIO, Beijing, China) at a 1:2,000 dilution for $1 \mathrm{~h}$ at room temperature after being washed. Reactive proteins were viewed by enhanced chemoluminescence (Applygen Technologies Inc.). The signals were detected with FujiFilm Las-3000 (Tokyo, Japan). The intensity of the detected bands was analyzed using the Image J program (National Institutes of Health, Bethesda, MD, USA).

Measurement of nicotinamide adenine dinucleotide phosphate $(N A D(P) H)$ oxidase activity in isolated glomeruli. The renal cortex was separated from the medulla. Cortical sections were placed into ice-cold RPMI-1640 medium (Sigma-Aldrich, St. Louis, MO, USA) and cut into three 2-mm sections. Glomeruli were isolated using the technique of differential sieving with stainless steel grids of 80,150 and 200 mesh size and then resuspended in RPMI-1640 medium (27). NAD(P)H oxidase activities in glomeruli were measured using tiron-inhibitable lucigenin chemiluminescence, as previously described (28).

Statistical analysis. All data are expressed as the mean \pm standard deviation. Statistical comparisons were performed using Student's t-test, except the histological analyses in interstitium-tubular lesions, which were analyzed by Pearson's $\chi^{2}$ test. SPSS version 19.0 software (SPSS Inc., Chicago, IL, USA) was used to perform the Student's t-test. $\mathrm{P}<0.05$ was considered to indicate a statistically significant difference.

\section{Results}

Effect of NBP on blood pressure. Prior to drug treatment, the average systolic pressure (SAP) of SHRs was $200 \mathrm{mmHg}$,
$\mathbf{A}$

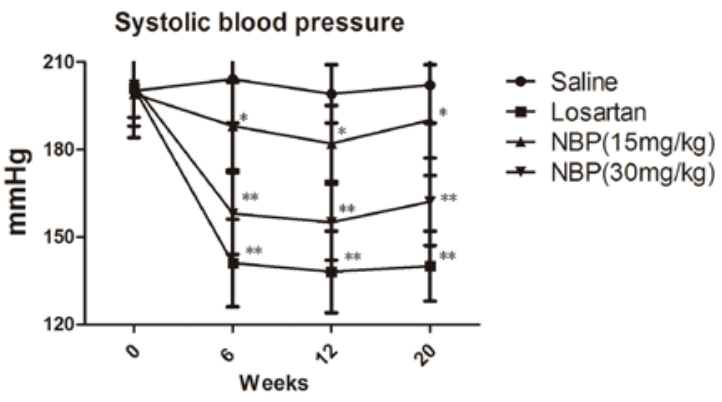

$\mathbf{B}$

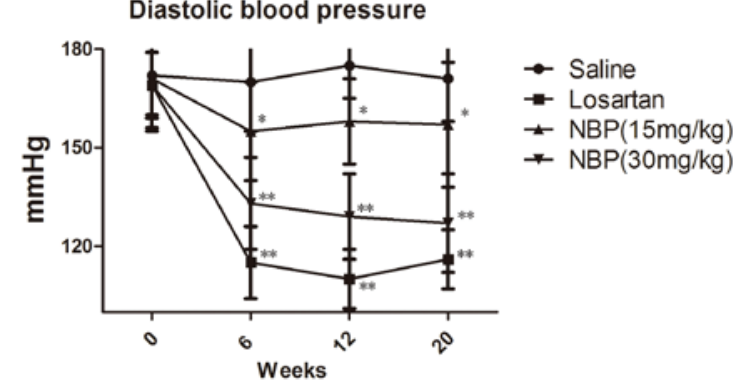

Figure 1. Effect of NBP and losartan on (A) average systolic blood pressure and (B) average diastolic blood pressure in SHRs over 20 weeks of administration. ${ }^{*} \mathrm{P}<0.05$ or ${ }^{* * *} \mathrm{P}<0.01$ for losartan or NBP groups compared with the saline group at all time points. NBP, 3-n-butylphthalide; SHRs, spontaneously hypertensive rats.

$56 \mathrm{mmHg}$ higher than that of normotensive rats (data not shown) and the average diastolic pressure (DAP) was $172 \mathrm{mmHg}$, $50 \mathrm{mmHg}$ higher than that of normotensive rats (data not shown).

Following drug treatment, blood pressure was measured every four weeks. The results are shown in Fig. 1A and B. Compared with the saline control group, SHRs in the NBP treatment groups showed lower SAP and DAP, and this effect occurred in a dose-dependent manner $(\mathrm{P}<0.05)$. However, the blood pressure-lowering effect of NBP was less marked than that of losartan.

Benefits of NBP on renal function. Table 1 shows the results of the effects of NBP and losartan on the levels of BUN, CCr and urinary albumin excretion (UAE) in SHRs. Compared with normotensive rats, these three parameters were initially above the normal ranges (UAE, $20.57 \pm 4.92 \mathrm{mg} / \mathrm{ml} /$ day; BUN, $16.77 \pm 1.26 \mathrm{mg} / \mathrm{dl}$; and $\mathrm{CCr}, 1.61 \pm 0.37 \mathrm{ml} / \mathrm{min} / 100 \mathrm{~g}$ bdy weight). As shown in Table 1 , treatment with NBP at 15 and 


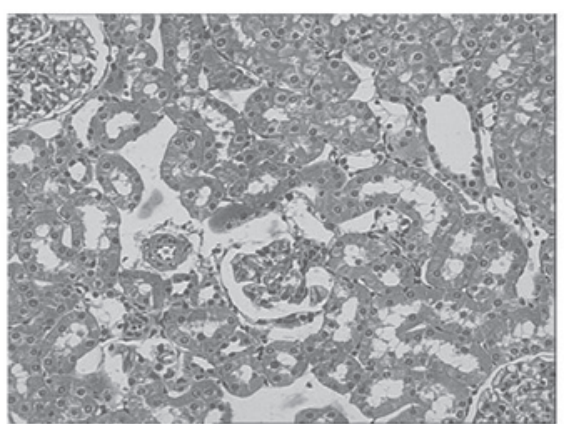

Saline control

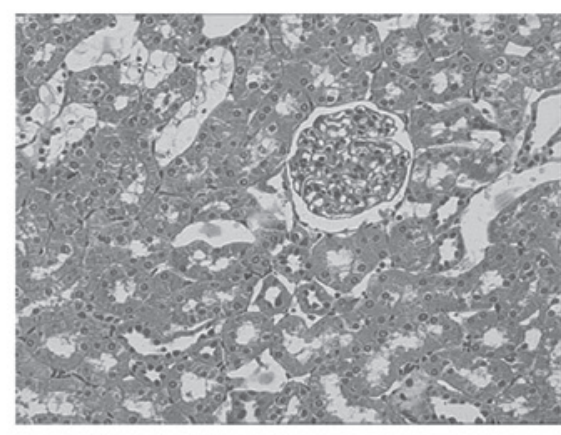

butylphthalide (30mg/kg)

B

H\&E (x100) tubule lesions

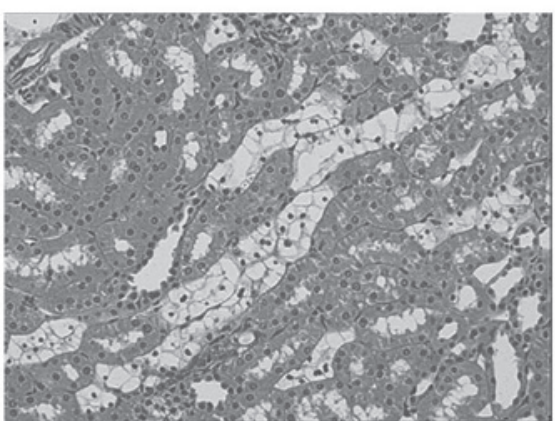

Saline control
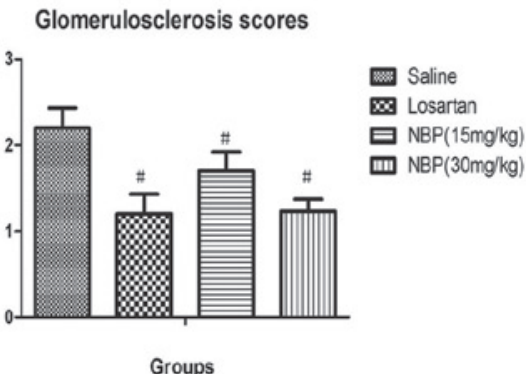

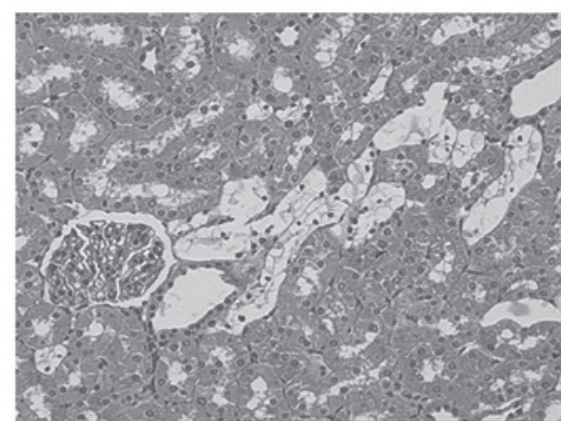

butylphthalide $(30 \mathrm{mg} / \mathrm{kg})$

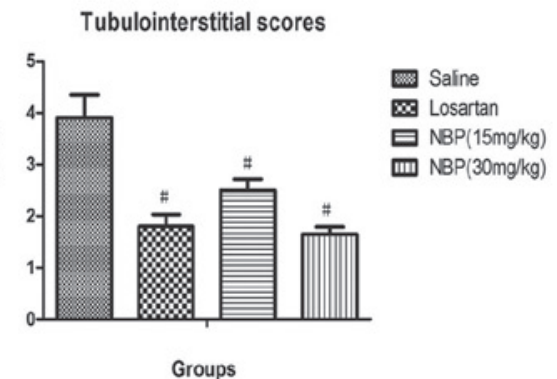

Figure 2. Representative images of H\&E staining tissue sections of (A) glomerulus lesions and (B) renal tubule lesions from kidneys collected from spontaneously hypertensive rats in the saline control and NBP $30 \mathrm{mg} / \mathrm{kg}$ treatment groups. ${ }^{\#} \mathrm{P}<0.05$, compared with the control group. H\&E, hematoxylin and eosin; NBP, 3-n-butylphthalide.

$30 \mathrm{mg} / \mathrm{kg}$ significantly decreased the levels of BUN and UAE and increased the $\mathrm{CCr}$ compared with the saline control $(\mathrm{P}<0.05)$. Losartan produced similar effects.

Benefits of NBP on the histopathology of the kidneys. Histopathological findings in SHRs in the saline control and $30 \mathrm{mg} / \mathrm{kg} \mathrm{NBP}$ groups are shown in Fig. 2. In the absence of drug treatment, SHRs exhibited greater degrees of glomerulosclerosis and apparent histological abnormalities in the interstitium-tubule areas, including interstitium infiltration of inflammatory cells, fibrosis, tubular dilatation and protein casts.

NBP treatment over 20 weeks resulted in significant alleviation of glomerulosclerosis $(\mathrm{P}<0.05)$. At doses of 15 and $30 \mathrm{mg} / \mathrm{kg}$, reduction in glomeruloscerosis scores was significant compared with the control group $(\mathrm{P}<0.05)$. Furthermore, at each dose, NBP significantly reduced interstitium infiltration of inflammatory cells, fibrosis, tubular dilatation and protein casts in SHRs, which was observed in the histopathological sections and was objectively calculated using the tubulointerstitial scoring system $(\mathrm{P}<0.05)$.

Expression of TNF- $\alpha, I L-6$ and $N F-\kappa B$ by IHC. As shown in Fig. 3, immunohistochemistry demonstrated positive staining of TNF- $\alpha$, IL- 6 and NF- $\kappa$ B in glomeruli and tubules of all the groups. NBP decreased the expression of these cytokines and chemokines in glomeruli and tubules in a dose-dependent manner compared with the saline control group.

TGF- $\beta 1$ protein contents in blood and renal tissues. ELISA and western blotting confirmed high expression of TGF- $\beta 1$ in peripheral blood and renal tissues of SHRs treated with saline, 
A

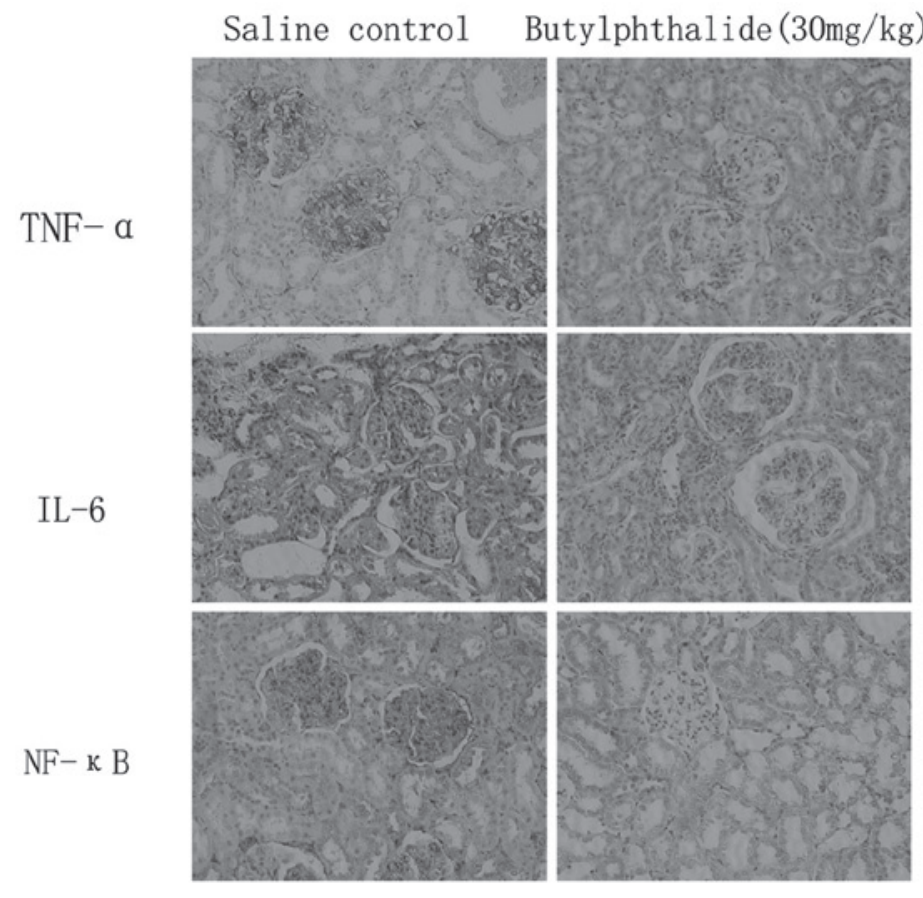

B

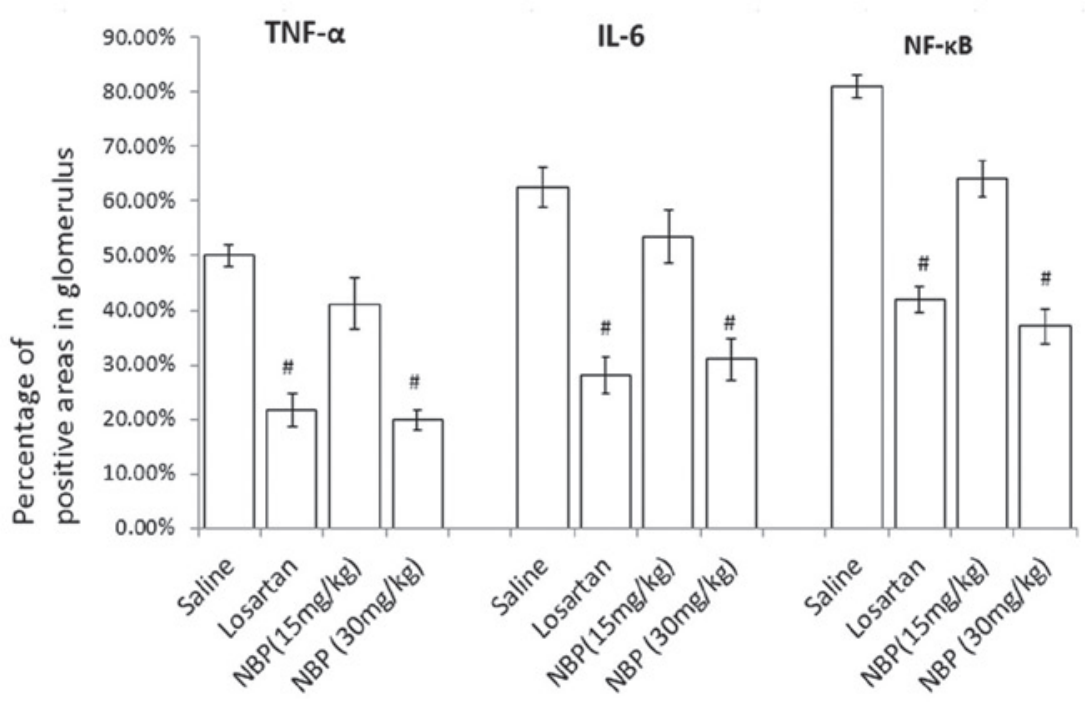

Figure 3. (A) Expression and (B) quantitation analysis of TNF- $\alpha$, IL-6 and NF- $\kappa$ B in the glomeruli, $\mathrm{x} 200$, designated by percentage of positive areas in glomerulus). ${ }^{*} \mathrm{P}<0.05,{ }^{*} \mathrm{P}<0.01$, compared with the control group. NBP 3-n-butylphthalide; TNF- $\alpha$, Tumor necrosis factor- $\alpha$; IL-6, interleukin 6; NF- $\mathrm{BB}$, nuclear factor-кB.

suggesting that TGF- $\beta 1$ may be involved in the development of fibrosis in hypertensive nephropathy.

As shown in Fig. 4, the blood concentration of TGF- $\beta 1$ was significantly reduced in NBP- and losartan-treated rats, compared with saline control animals. Western blotting showed that the expression of TGF- $\beta 1$ in kidney tissues was also reduced by NBP treatment, in a dose-dependent manner (Fig. 5). These findings suggested that NBP may slow renal fibrosis via modulation of TGF- $\beta 1$ signaling pathways.

$N B P$ treatment reduced $N A D(P) H$ oxidase activity in isolated glomeruli. The $\mathrm{NAD}(\mathrm{P}) \mathrm{H}$ oxidase activity in isolated glomeruli of the three treatment groups was significantly lower than the saline SHR group, which suggested NBP and losartan may reduce the oxidative stress in the glomeruli of hypertensive rats (Fig. 6).

\section{Discussion}

NBP has shown beneficial effects in the treatment of hypertension, stroke and cerebral ischemia in clinical practice (13). It was hypothesized that NBP may also be effective in the treatment of hypertensive nephropathy. The current study used SHRs as an animal model in order to test this hypothesis.

In the present study, SHRs aged 16 weeks were used. At the end of the 20 week treatment period, all rats progressed to chronic renal dysfunction, with low creatinine clearance, high UAE and high BUN levels. NBP significantly attenuated renal dysfunction and improved these parameters of renal function, as demonstrated by biochemical analysis. NBP treatment at $30 \mathrm{mg} / \mathrm{kg}$ dosage had a similar effect on improving biochemical parameters of renal function as losartan treatment at $10 \mathrm{mg} / \mathrm{kg}$ dosage. 


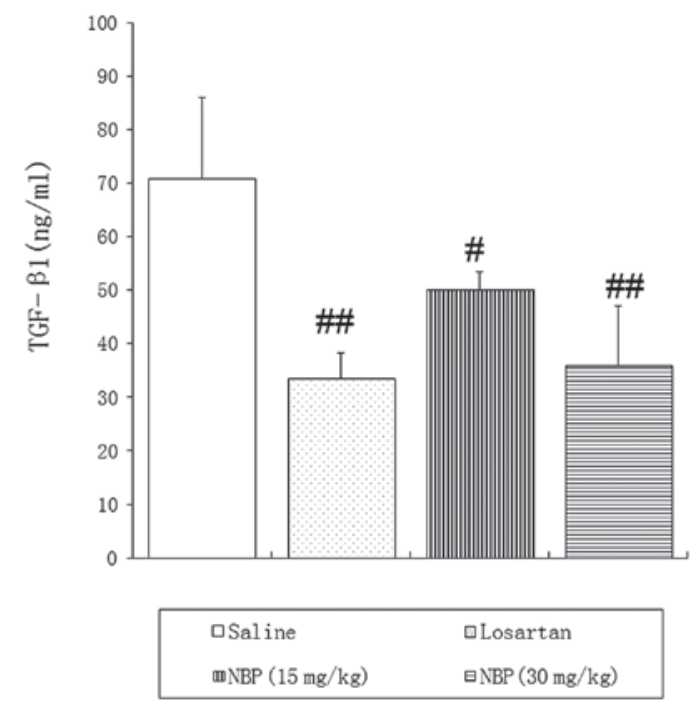

Figure 4. Enzyme-linked immunosorbent assay analysis of TGF- $\beta 1$ levels in blood serum after administration over 20 weeks. ${ }^{\#} \mathrm{P}<0.05$ and ${ }^{\# \#} \mathrm{P}<0.01$, compared with the control group. TGF- $\beta 1$, transforming growth factor- $\beta 1$; NBP, 3-n-butylphthalide.

A
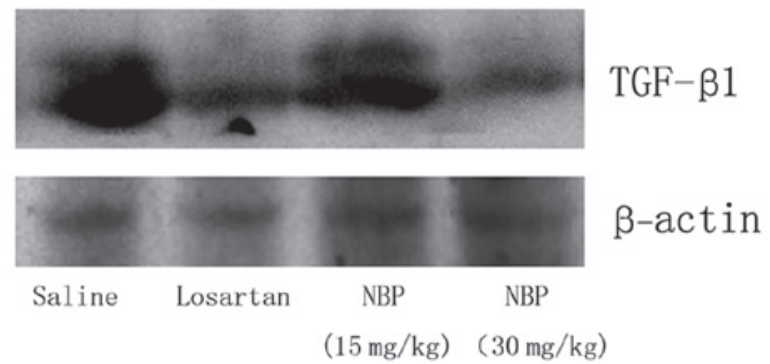

B

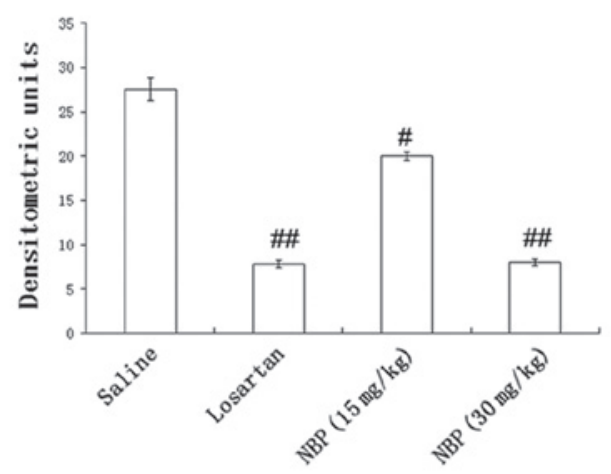

Figure 5. TGF- $\beta 1$ protein levels in kidneys of spontaneously hypertensive rats. (A) Western blot of TGF- $\beta 1$ in kidney tissues lysates. Kidney tissues lysates $(60 \mu \mathrm{g})$ were used in SDS-PAGE in each lane. Each lane represents a pool of kidney tissues from 10 rats per group. (B) Densitometric analysis of TGF- $\beta 1$ protein levels. ${ }^{\#} \mathrm{P}<0.05$ and ${ }^{\# \#} \mathrm{P}<0.01$, compared with the control group. TGF- $\beta 1$, transforming growth factor- $\beta 1$; NBP, 3-n-butylphthalide.

Notably, histopathological analysis showed that NBP significantly alleviated injuries in glomeruli and proximal tubules. The glomerulosclerosis scores in the NBP-treated SHRs were significantly lower than those in the control SHRs. The degree of tubulointerstitial fibrosis and other tubular-interstitium injuries (interstitium infiltration, interstitium fiborosis, tubular dilatation and tubule-interstitium protein casts) was also significantly reduced after NBP treat-

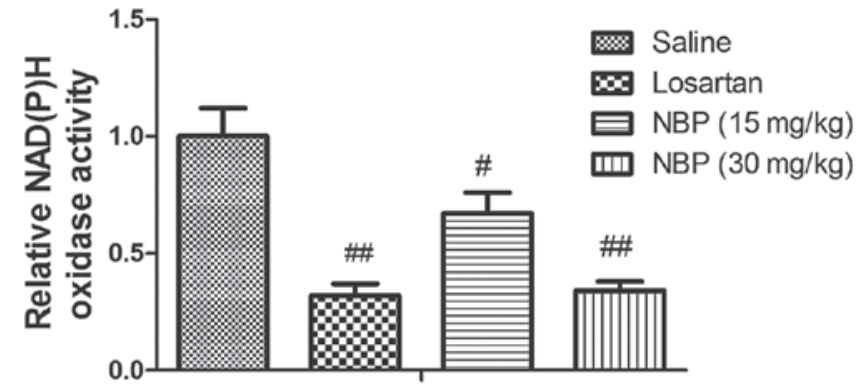

Groups

Figure 6. NAD $(\mathrm{P}) \mathrm{H}$ oxidase activity in isolated rat glomeruli. ${ }^{*} \mathrm{P}<0.05$ and ${ }^{\# \#} \mathrm{P}<0.01$, compared with the control group. NBP, 3-n-butylphthalide; $\mathrm{NAD}(\mathrm{P}) \mathrm{H}$, nicotinamide adenine dinucleotide phosphate.

ment $(\mathrm{P}<0.05)$. These results suggest the therapeutic potential of NBP in the treatment of hypertensive nephropathy.

TGF- $\beta 1$ and its receptors are important in end-stage renal fibrosis $(22,29)$. Their over-activation is known to enhance the progression of renal fibrosis. In the present study, ELISAs and western blotting demonstrated significantly attenuated expression of TGF- $\beta 1$ protein in the blood and kidney tissues of NBP-treated SHRs, suggesting one possible mechanism underlying NBP suppression of hypertensive nephropathy.

In terms of mechanisms underlying the renal protective effect of NBP in hypertensive nephropathy, it was hypothesized that the blood pressure lowering effect of this compound was one of the most significant. The results demonstrated that NBP decreased the SAP and DAP significantly in SHRs during long-term treatment. Thus, reducing BP is an important mechanism by which NBP may slow the progression of hypertensive nephropathy. This has also been demonstrated in previous studies $(6,7)$.

Inflammatory cytokines have been shown to be pivotal in the pathogenesis of hypertension associated with end-stage renal disease $(30,31)$. The present study provides strong evidence for an anti-inflammatory effect of NBP in the kidney. The results suggest that NBP may exert such a role by inducing normalization of the levels of pro-inflammatory cytokines (TNF- $\alpha$ and IL-6) together with a reduction in levels of the transcription factor NF- $\mathrm{KB}$. It was shown that NBP inhibited the NF- $\kappa$ B pathway together with a downregulation of the expression of several proinflammatory cytokine genes. These included IL-6, which causes alterations in endothelial permeability, induction of mesangial cell proliferation and increased fibronectin expression in chronic nephropathy $(32,33)$. Proinflammatory mediators and increasing oxidative stress are important in the pathogenesis of hypertensive nephrology (34).

Increasing oxidative stress is considered to contribute to progress of hypertensive nephropathy in the glomeruli. $\mathrm{NAD}(\mathrm{P}) \mathrm{H}$ oxidase is a major source of reactive oxygen species. Oxidative stress depletes NO and causes endothelial dysfunction, which initiates arteriosclerosis (35). In the current study, NBP significantly decreased NADP(H) oxidase activity in the glomeruli, which may protect endothelial function and thus offer a significant clinical benefit. 
In conclusion, the present study showed that chronic administration of NBP normalizes SAP and DAP, improves proteinuria and ameliorates fibrosis in the kidney of SHRs. These effects were associated with a reduction in renal fibrosis and oxidative stress, and decreased levels of TNF- $\alpha$, IL- 6 and $\mathrm{NF}-\kappa \mathrm{B}$. This study provides strong evidence for a protective role of NBP in the kidney.

\section{Acknowledgements}

The authors would like to acknowledge the support provided by the Tianjin Medical University General Hospital, Tianjin, China.

\section{References}

1. Azegami T, Sasamura H, Hayashi K and Itoh H: Vaccination against the angiotensin type 1 receptor for the prevention of L-NAME-induced nephropathy. Hypertens Res 35: 492-499, 2012.

2. Hill GS: Hypertensive nephrosclerosis. Curr Opin Nephrol Hypertens 17: 266-270, 2008.

3. Zhang S, Li H, LiY, et al: Nicousamide normalizes renovascular hypertension in two-kidney one-clip hypertensive rats. Biomed Rep 1: 89-92, 2013.

4. Zhang S, Li Y, Li H, et al: Renal protective effect of nicousamide on hypertensive nephropathy in spontaneously hypertensive rats. Biomed Rep 1: 34-40, 2013

5. Iseki K: Factors influencing the development of end-stage renal disease. Clin Exp Nephrol 9: 5-14, 2005.

6. Klag MJ, Whelton PK, Randall BL, et al: Blood pressure and end-stage renal disease in men. N Engl J Med 334: 13-18, 1996.

7. Tozawa M, Iseki K, Iseki C, Kinjo K, Ikemiya Y and Takishita S: Blood pressure predicts risk of developing end-stage renal disease in men and women. Hypertension 41: 1341-1345, 2003.

8. Chen Y, Lipkowitz MS, Salem RM, et al: Progression of chronic kidney disease: Adrenergic genetic influence on glomerular filtration rate decline in hypertensive nephrosclerosis. Am J Nephrol 32: 23-30, 2010.

9. Wang G, Lai FM, Kwan BC, et al: Expression of ACE and ACE2 in patients with hypertensive nephrosclerosis. Kidney Blood Press Res 34: 141-149, 2011.

10. Moghadam MH, Imenshahidi $M$ and Mohajeri SA: Antihypertensive effect of celery seed on rat blood pressure in chronic administration. J Med Food 16: 558-563, 2013.

11. Zhang L, Lü L, Chan WM, et al: Effects of DL-3-n-butylphthalide on vascular dementia and angiogenesis. Neurochem Res 37 911-919, 2012.

12. Liao SJ, Lin JW, Pei Z, et al: Enhanced angiogenesis with dl-3n-butylphthalide treatment after focal cerebral ischemia in RHRSP. Brain Res 1289: 69-78, 2009.

13. Chong ZZ and Feng YP: dl-3-n-butylphthalide attenuates reperfusion-induced blood-brain barrier damage after focal cerebral ischemia in rats. Zhongguo Yao Li Xue Bao 20: 696-700, 1999.

14. Cui LY, Zhu YC, Gao S, et al: Ninety-day administration of dl-3-n-butylphthalide for acute ischemic stroke: a randomized, double-blind trial. Chinese Med J (Engl) 126: 3405-3410, 2013

15. Li L, Zhang B, Tao Y, et al: DL-3-n-butylphthalide protects endothelial cells against oxidative/nitrosative stress, mitochondrial damage and subsequent cell death after oxygen glucose deprivation in vitro. Brain Res 1290: 91-101, 2009.

16. Liu CL, Liao SJ, Zeng JS, et al: dl-3n-butylphthalide prevents stroke via improvement of cerebral microvessels in RHRSP. J Neurol Sci 260: 106-113, 2007.
17. Tian D, Ling S, Chen G, et al: Hypertensive nephropathy treatment by heart-protecting musk pill: a study of anti-inflammatory therapy for target organ damage of hypertension. Int J Gen Med 4: 131-139, 2011.

18. Sun L, Ke Y, Zhu CY, et al: Inflammatory reaction versus endogenous peroxisome proliferator-activated receptors expression, re-exploring secondary organ complications of spontaneously hypertensive rats. Chin Med J (Engl) 121: 2305-2311, 2008.

19. Koshikawa S, Nishikimi T, Inaba C, et al: Fasudil, a Rho-kinase inhibitor, reverses L-NAME exacerbated severe nephrosclerosis in spontaneously hypertensive rats. J Hypertens 26: 1837-1848, 2008.

20. Alfie J, Aparicio LS and Waisman GD: Current strategies to achieve further cardiac and renal protection through enhanced renin-angiotensin-aldosterone system inhibition. Rev Recent Clin Trials 6: 134-146, 2011.

21. Berl T: Review: renal protection by inhibition of the renin-angiotensin-aldosterone system. J Renin Angiotensin Aldosterone Syst 10: 1-8, 2009.

22. Zhang S, Xin H, Li Y, et al: Skimmin, a coumarin from Hydrangea paniculata, slows down the progression of membranous glomerulonephritis by anti-inflammatory effects and inhibiting immune complex deposition. Evid Based Complement Alternat Med: 819296, 2013

23. Tapia E, Sanchez-Lozada LG, Soto V, et al: Sildenafil treatment prevents glomerular hypertension and hyperfiltration in rats with renal ablation. Kidney Blood Press Res 35: 273-280, 2012.

24. Pörsti I, Fan M, Kööbi P, et al: High calcium diet down-regulates kidney angiotensin-converting enzyme in experimental renal failure. Kidney Int 66: 2155-2166, 2004.

25. Li P, Ma LL, Xie RJ, et al: Treatment of 5/6 nephrectomy rats with sulodexide: a novel therapy for chronic renal failure. Acta Pharmacol Sin 33: 644-651, 2012.

26. Giani JF, Muñoz MC, Pons RA, et al: Angiotensin-(1-7) reduces proteinuria and diminishes structural damage in renal tissue of stroke-prone spontaneously hypertensive rats. Am J Physiol Renal Physiol 300: F272-F282, 2011.

27. Tomohiro T, Kumai T, Sato T, et al: Hypertension aggravates glomerular dysfunction with oxidative stress in a rat model of diabetic nephropathy. Life Sci 80: 1364-1372, 2007.

28. Li L, Yang R, Sun K, et al: Cerebroside-A provides potent neuroprotection after cerebral ischaemia through reducing glutamate release and $\mathrm{Ca}^{2+}$ influx of NMDA receptors. Int J Neuropsychopharmacol 15: 497-507, 2012.

29. Liu Y: Renal fibrosis: new insights into the pathogenesis and therapeutics. Kidney Int 69: 213-217, 2006.

30. Tomino Y, Hagiwara S and Gohda T: AGE-RAGE interaction and oxidative stress in obesity-related renal dysfunction. Kidney Int 80: 133-135, 2011.

31. Koyner JL, Sher Ali R and Murray PT: Antioxidants. Do they have a place in the prevention or therapy of acute kidney injury? Nephron Exp Nephrol 109: e109-e117, 2008.

32. Semedo P, Correa-Costa M, Antonio Cenedeze M, et al: Mesenchymal stem cells attenuate renal fibrosis through immune modulation and remodeling properties in a rat remnant kidney model. Stem Cells 27: 3063-3073, 2009.

33. Azuma H, Nadeau K, Takada M, Mackenzie HS and Tilney NL: Cellular and molecular predictors of chronic renal dysfunction after initial ischemia/reperfusion injury of a single kidney. Transplantation 64: 190-197, 1997.

34. Navarro-González JF and Mora-Fernández C: The role of inflammatory cytokines in diabetic nephropathy. J Am Soc Nephrol 19: 433-442, 2008.

35. Cervenka L and Heller J: Comparison of the effects of a low-protein diet with the effects of a converting enzyme inhibitor on the progression of renal insufficiency in hypertensive rats. Renal Fail 18: 173-180, 1996. 